\title{
Experiences integrating and using laser educational kits at Irvine Valley College's laser technology program
}

Donn Silberman, Gabriel Blanks, Stefan Forschner, Brian Monacelli, Desiré Whitmore, et al.

Donn M. Silberman, Gabriel Blanks, Stefan Forschner, Brian Monacelli, Desiré Whitmore, Debbie Donahue, "Experiences integrating and using laser educational kits at Irvine Valley College's laser technology program," Proc. SPIE 10741, Optics Education and Outreach V, 107410G (14 September 2018); doi: $10.1117 / 12.2322490$

EDIE Event: SPIE Optical Engineering + Applications, 2018, San Diego, California, United States 


\title{
Experiences integrating and using laser educational kits at Irvine Valley College's laser technology programs
}

\author{
a,b,c Donn M. Silberman, ${ }^{\mathrm{a}, \mathrm{c}, \mathrm{d}}$ Gabriel Blanks, ${ }^{\mathrm{a}, \mathrm{d}}$ Stefan Forschner, ${ }^{\mathrm{d}}$ Brian Monacelli, ${ }^{\mathrm{d}, \mathrm{e}}$ Desiré \\ Whitmore, ${ }^{\mathrm{a}}$ Debbie Donahue \\ ${ }^{\mathrm{a}}$ eLas Americas (United States), ${ }^{\mathrm{b}}$ Optics Institute of Southern California (United States) \\ ${ }^{\mathrm{c}}$ Univ. of California, Irvine (United States), ${ }^{\mathrm{d}}$ Irvine Valley College (United States), \\ ${ }^{\mathrm{e}}$ Exploratorium (United States)
}

\begin{abstract}
Irvine Valley College was the first school in the United States to have both HeNe and Nd:YAG open-cavity laser educational kits from eLas (formerly PI miCos Campus) successfully integrated into their extensive hands-on Laser Technology program. This paper is presented from two students' perspective, describing the students' experiences and including comments from the professors who integrated the laser kits into the curriculum. The main benefits these laser education kits provide for both students and faculty include their specific industrial-quality design for intensive hands-on education. Students learn about laser components and the techniques required to align a laser cavity. Theses laser systems come with lesson plans and experiments that faculty may use as is, or modify to suit a particular emphasis in their curriculum. Once alignment is achieved on a repeatable basis, then many experiments can be performed successfully, such as studying laser mode structure, input current versus output power, and wavelength stability.
\end{abstract}

Keywords: Photonics education, laser education kits, student testimonials, hands-on laser education

\section{Introduction and History}

Irvine Valley College (IVC) has a long history teaching optical and laser technology that goes back over a uarter of a century. Various incarnations of the programs have ebbed and flowed with support from local industry and the Optical Society of Southern California, given the industry's dynamic needs for trained technical employees. VC has partnered with other local educational programs such as those at the University of California, Irvine, most otably the Beckman Laser Institute and Medical Clinic, to provide well-educated employees to the local optics industry. In the early 1990s, the IVC Laser and Electro-optics Technology (LET) program had a classroom and teaching laboratory complete with various lasers, optical, electrical, and optomechanical hardware on the main campus and was so integrated into the fabric of the college that they named their mascot the "Lasers". (Reference www.ivclasers.com) Figure 1 is a photograph of the IVC gymnasium wall during a STEM event with their Laser logo beneath the name of the college. In the mid-2000's, IVC obtained a grant through the Center for Applied Competitive Technologies (CACT) and had a classroom, lab, offices and other spaces in a temporary facility known as CACT Photonics at Advanced Technology and Education Park (ATEP). Figure 2 is a photograph of an instructor

Optics Education and Outreach V, edited by G. Groot Gregory, Proc. of SPIE Vol. 10741, 107410G

(c) 2018 SPIE · CCC code: 0277-786X/18/\$18 · doi: 10.1117/12.2322490 
and some students outside the building. From the late-2000's to mid-2010's, the Laser Electro-Optics Technology program was housed at a campus in Tustin, California, with even more space, equipment, and students, as shown in Figures 3 through 5.

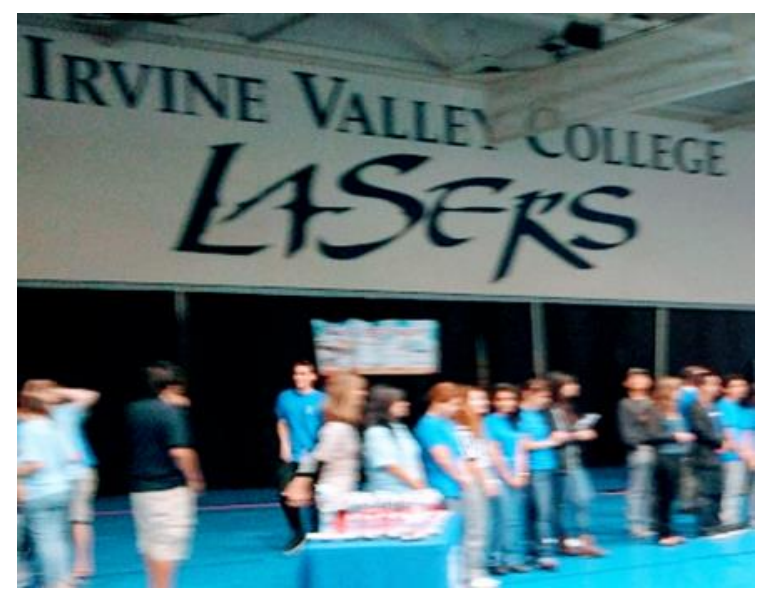

Fig. 1. IVC gymnasium wall during a STEM event with their Laser logo underneath the name of the college.

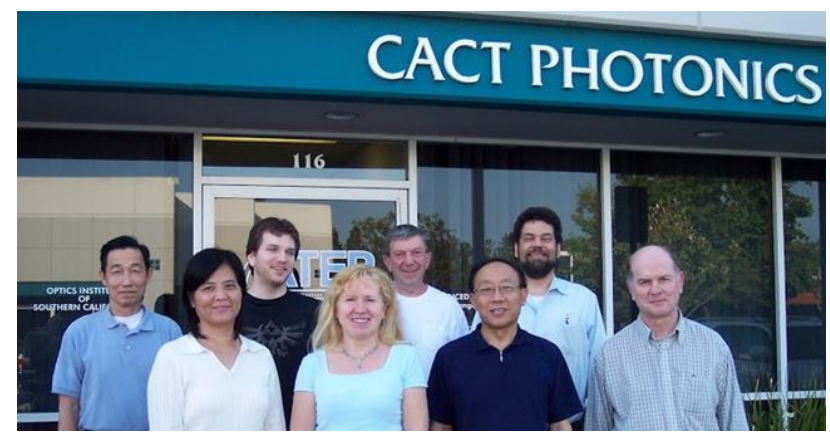

Fig. 2 is a photograph of an instructor and some students outside the CACT Photonics building in 2006.

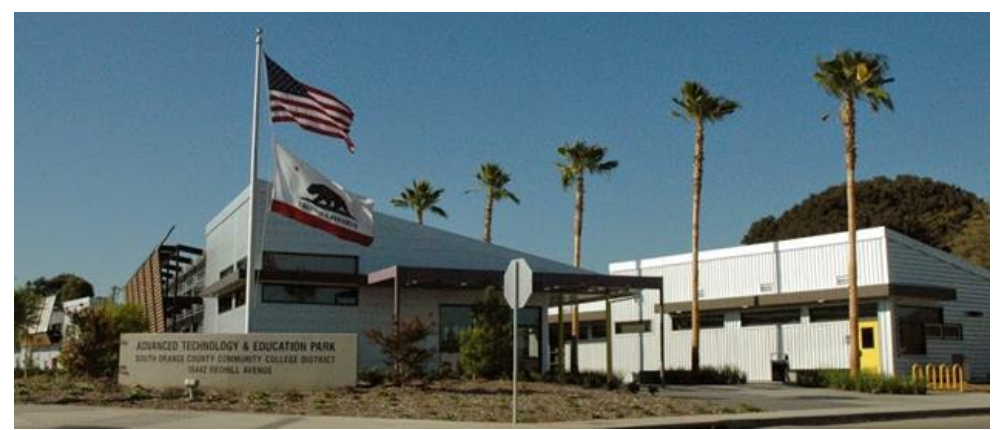

Fig. 3 is a photograph of IVC ATEP Campus in 2007. This was the location of the Laser Electro-optics Technology Program for 11 years. 


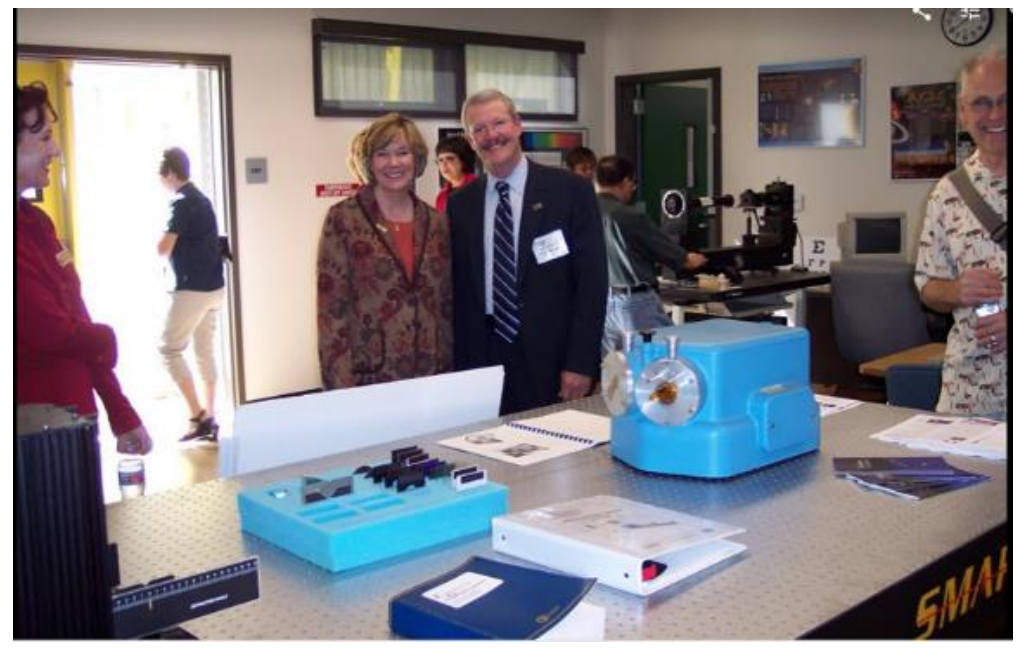

Fig. 4 is a photograph of IVC President Glenn Roquemore and his wife during the grand opening of the campus in 2007 . He has been strong supporter of the Laser programs for many years.

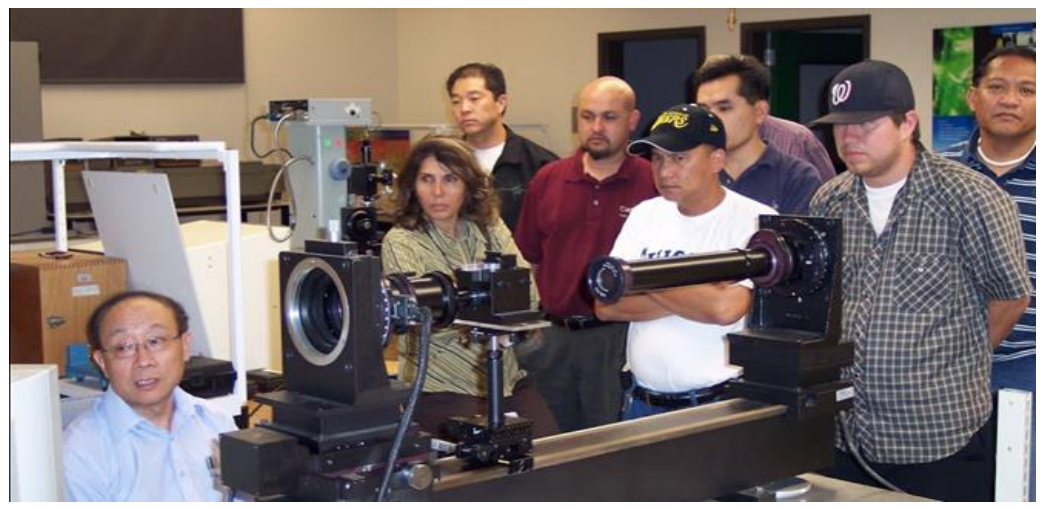

Fig. 5 is a photograph of IVC ATEP students in 2007. At this time the focus was of the Laser Electro-optics Technology Program was more on optics. Here they are learning about optical performance testing using an MTF Bench. 
About five years ago, two educational open-cavity laser kits were lent by PI miCos to IVC's Laser Technology program to help teach laser fundamentals. PI miCos offered these for sale to other colleges and universities in the US, and they assisted OP-TEC partner colleges in integrating the Nd:YAG Laser Kits into their Fundamentals of Laser Technology course. In 2016, these educational laser kits transitioned from PI miCos to eLas and eLas Americas, so that they could continue to be offered directly to schools. Since then, more optical technology programs have adopted these kits to help students learn about laser technology and the applied techniques required to align and maintain laser functionality. Figure 6 is a recent excerpt of the website for the program and includes a photograph of a student aligning the open-cavity HeNe laser from the kit.

\section{Introduction and History} Laser Electro-Optics at Irvine Valley College

\section{IRVINE VALLEY COLLEGE Photonics}
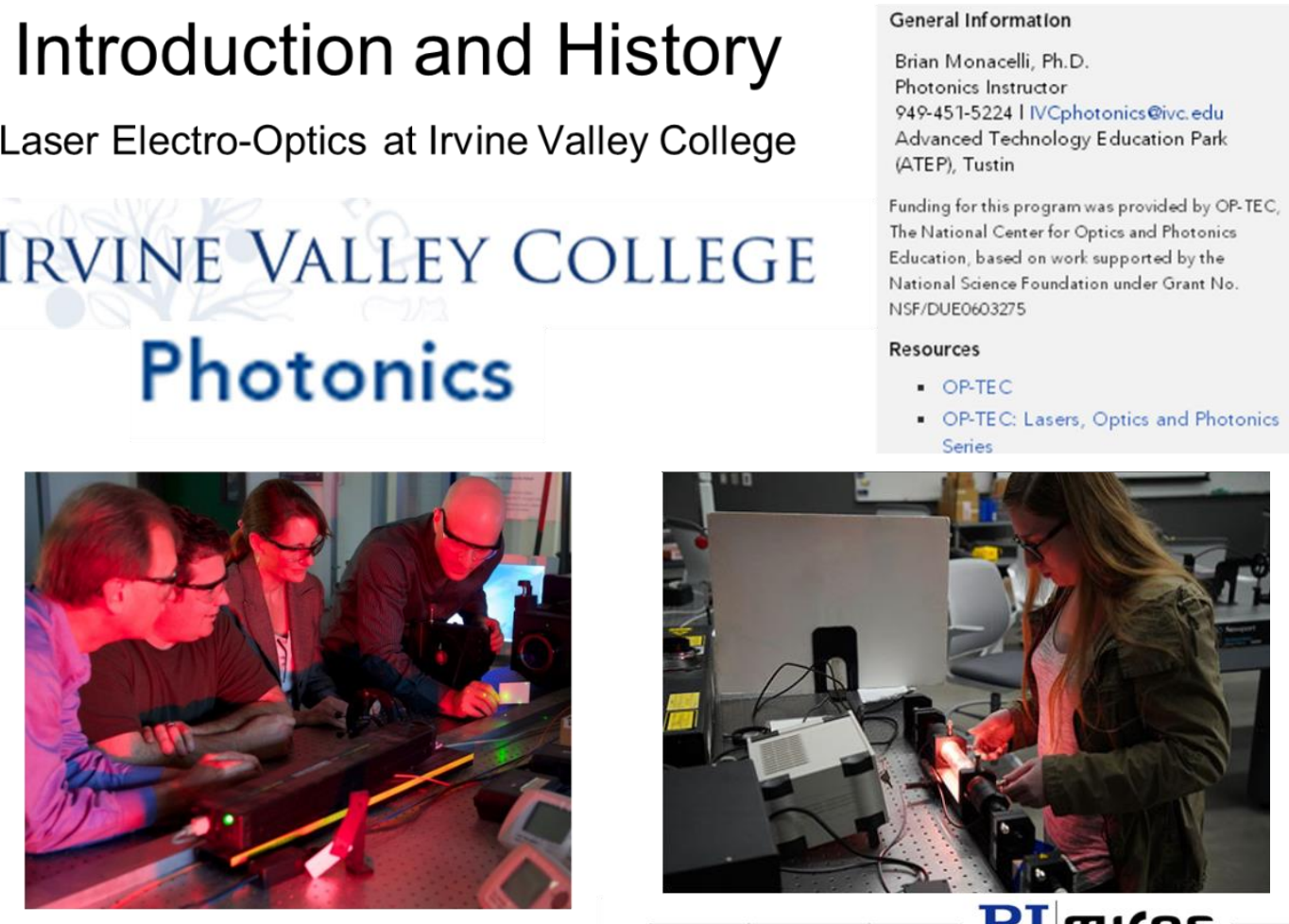

CA-1200 HeNe Laser

Fig. 6 is a modified image of the website home page for the program several years ago and includes a photograph of a student aligning the open cavity HeNe laser from the kit. 
The current program (http://academics.ivc.edu/idea/lasertech) at IVC is funded, in part, by a National Science Foundation Advanced Technology Education Grant, where Laser Technology instructors lead hands-on, laboratory-driven classes with state-of-the-art industrial equipment that was largely donated by its strong network of industrial partners. Instructors work with the local business and education community to provide shop-floor demonstrations to enrolled students and to increase public awareness of the diverse opportunities in the field of photonics. In addition to laboratory skills, students are also offered one-on-one career advice on topics such as résumé editing, LinkedIn profile building, and technical presentation skills.

This year, the new IVC Integrated Design, Engineering and Automation (IDEA) campus at ATEP began serving as a catalyst for innovative career education opportunities (https://atep.ivc.edu/). In the heart of Orange County, California, several complementary technical programs are offered alongside the Laser Technology program, including Design, Model Making, and Rapid Prototyping; Drafting and Engineering Technology; Electrical Technology; and Electronics Technology. The current website for this campus is shown in Figure 7 with open-cavity HeNe Laser system, professor and student shown prominently in the top left indicating the significance of this program to the college.

The importance of having these open-cavity laser system kits in the class laboratory is articulated by the students in the sections to follow. The remainder of this paper will be from the perspective of students and instructors who have gained extensive experience with these educational laser systems.

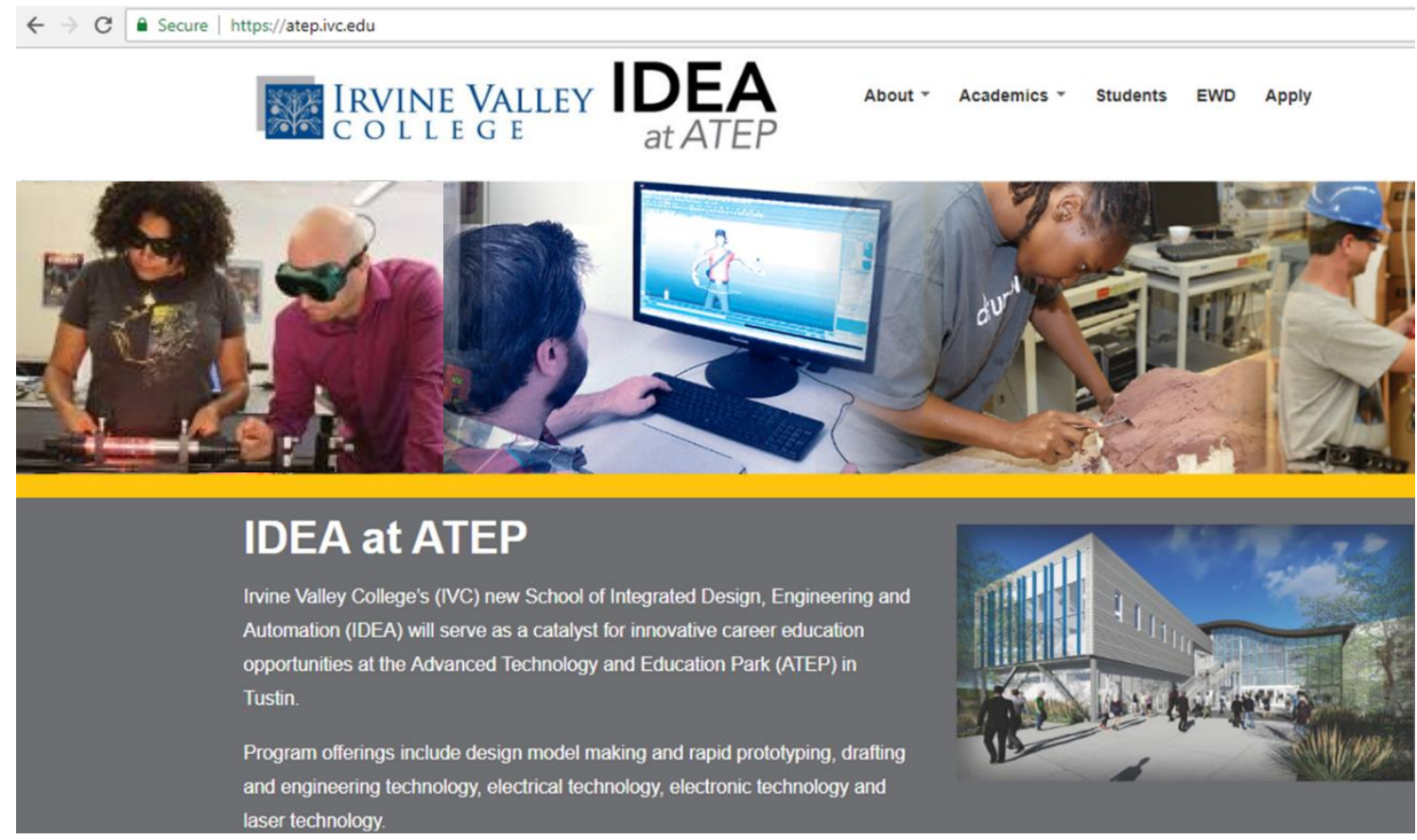

Fig. 7 is a modified image of the current website home page for IDEA at ATEP with the open cavity HeNe Laser system, two professors are shown prominently in the top left indicating the importance of this program to the college. 


\section{Stefan's experiences and perspectives on the technical benefits of the kits}

When I was first taking Irvine Valley College's Laser Technology Program, one of the experiments they had students do was align the HeNe and Nd:YAG open-cavity laser systems and write a report on their experience with the alignment process. For example, one of the biggest issues that I had when aligning the HeNe laser was the mirrors' optical surfaces not being clean enough to lase. This proved to be a very good lesson to me with attention to detail, had we not checked the surfaces of the mirrors, I would have continued to try and try again with realigning the mirrors and gain medium, only to yield no results. Figure 8 shows a photograph of the HeNe Laser kit and Figure 9 shows a diagram and descriptions of all the various components. One student in our program had a similar experience to mine and wrote in his lab report:

"Depending on whether you actually achieve lasing alignment or not, this could be one of the most rewarding or one of the most frustrating tasks. I fortunately had the opportunity of experiencing both the up and the down of this lab, and it taught me a valuable lesson of real life: that sometimes, even when we follow the directions to the letter, things aren't guaranteed to work, and the things we think are the problem don't always turn out to be the issue. That's why engineering work requires you to be a problem-solver with an open-mind and a stubborn heart that refuses to give up."

\section{elas}

\section{Helium Neon Laser (CA-1200)}

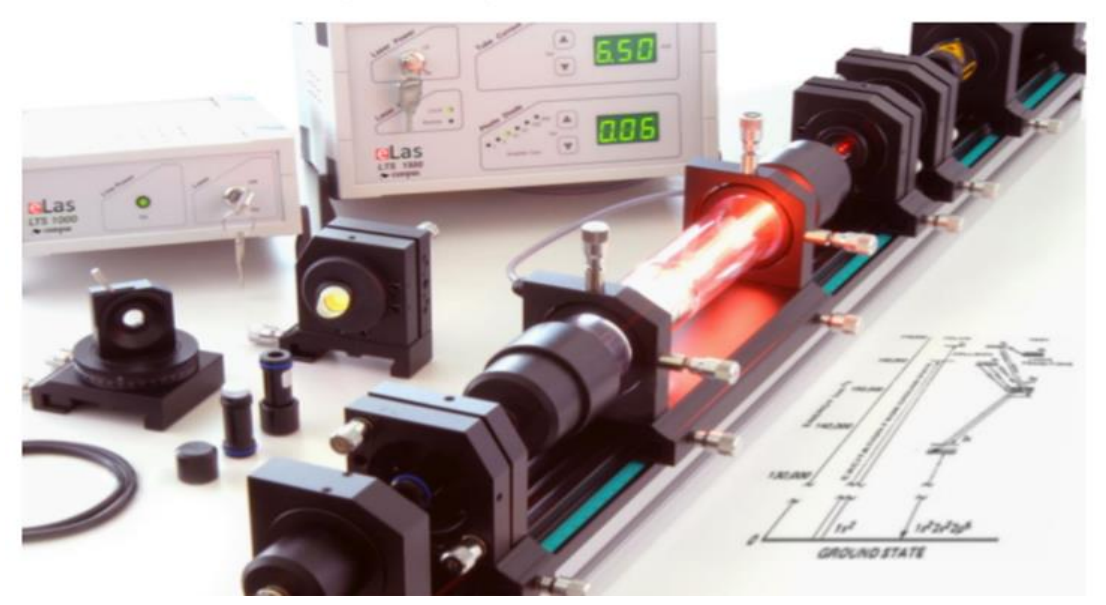

Fig. 8 shows a photograph of the eLas HeNe Laser kit and all the various components. 


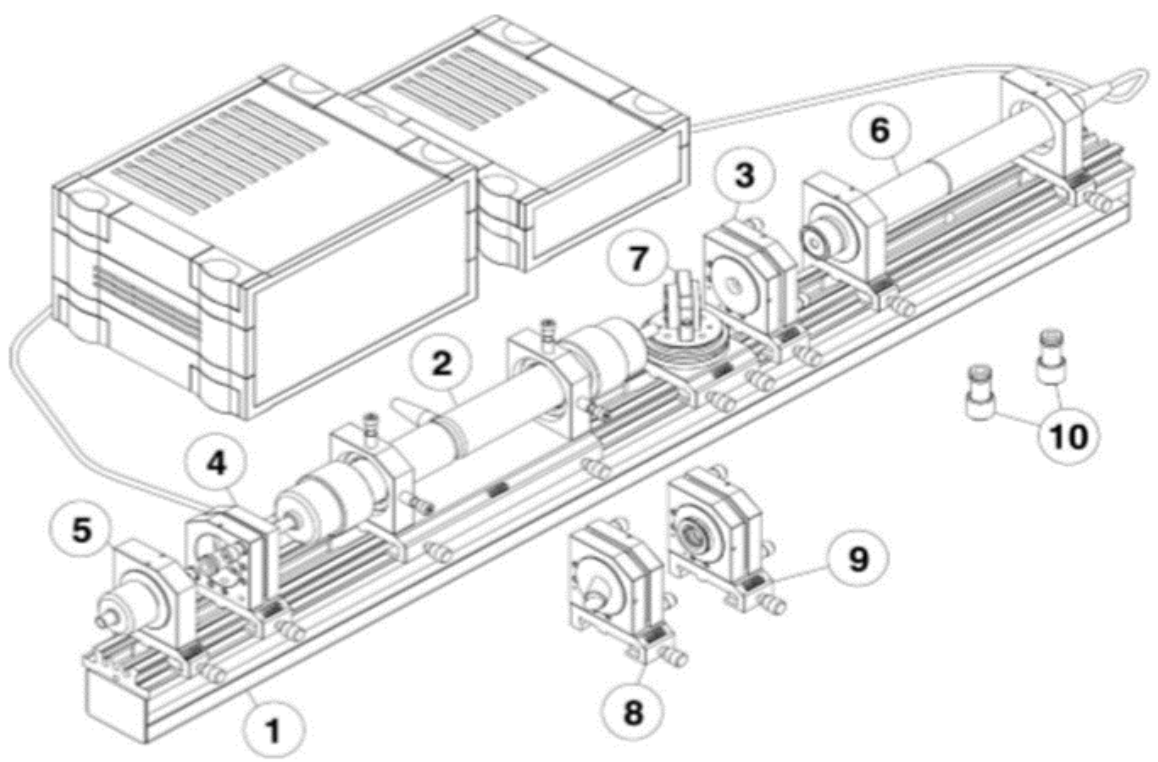

1 Flat rail $1500 \mathrm{~mm}$ reinforced by profile with scale

2 Controller LTS 1500 for HeNe tube and photo diode amplifier

3 Right kinematic mirror adjustment holder on carrier

4 Left kinematic mirror adjustment holder on carrier

5 Photo detector in holder on carrier with BNC cable

6 Adjustment laser (green DPSS module) in holder on carrier with power supply

7 Birefringent filter in rotating holder on carrier platform with rotating insert (Full and Extended Version)

8 Littrow prism in adjustable holder on carrier (Full and Extended Version)

9 Single mode etalon in adjustable holder on carrier (Full and Extended Version)

10 Set of 5 laser mirrors (3 mirrors in the Basic Version)

11 Optical transmission grating on carrier (Full and Extended Version, not shown)

12 Non-reflecting caliper (not shown)

13 Pair of polarization filters in rotational holders on carriers (Extended Version)

14 Half-wave plate in rotational holder on carrier (Extended Version)

15 Pair quarter-wave plates in rotational holders on carriers (Extended Version)

16 Set for optics cleaning (not shown)

17 User manual (not shown)

Fig. 9 shows a diagram with descriptions of all the various components. 
Fortunately for me, once that issue was dealt with, it was straightforward to align the HeNe Laser using the methods I was taught. This was partially due to the industrial-quality design of the parts in the open-cavity laser. A great example of this is how all of the parts connect to the same commercial-grade optical rail, so when you are aligning each part to the rest of the system, you do not have to worry about the parts being positioned off axis. Along with integrating the optical rail in the design, giving each of the mirrors knobs for precise angular alignment lets students focus on the specific techniques they develop using this system — without having to worry about developing hardware skills at the same time. Even the gain medium is mounted in a professional, student-friendly manner. With two separate points with axis adjustments, getting the gain medium aligned with the rest of the system is not difficult. When I was practicing aligning the gain medium, I mounted a negative lens to expand the alignment beam after leaving the gain medium, just to get a better idea of how the beam was being aberrated when going through the gain medium at an awry angle. Once the gain medium was properly aligned, I adjusted actuators of the gain medium to vertically and horizontally translate the gain medium for maximum power output.

While the HeNe laser system removes the hassle with learning alignment skills — allowing students to focus on technique - the best way to refine those skills would be to add some difficulty and nuance to put the students' skills to the test. Our way of testing a student's alignment skills at IVC was to have them align the Nd:YAG laser. Even though alignment between the HeNe and Nd:YAG is similar in concept, the added challenge of the Nd:YAG comes with working on optimizing an infrared laser beam. That, paired with an extra alignment with a frequencydoubling crystal, gives the Nd:YAG laser system its sense of achievement once the system is fully aligned. Figure 10 is a photograph of Stefan setting up the components of Nd:YAG laser system. The system is not yet activated since he is not wearing his laser safety glasses.

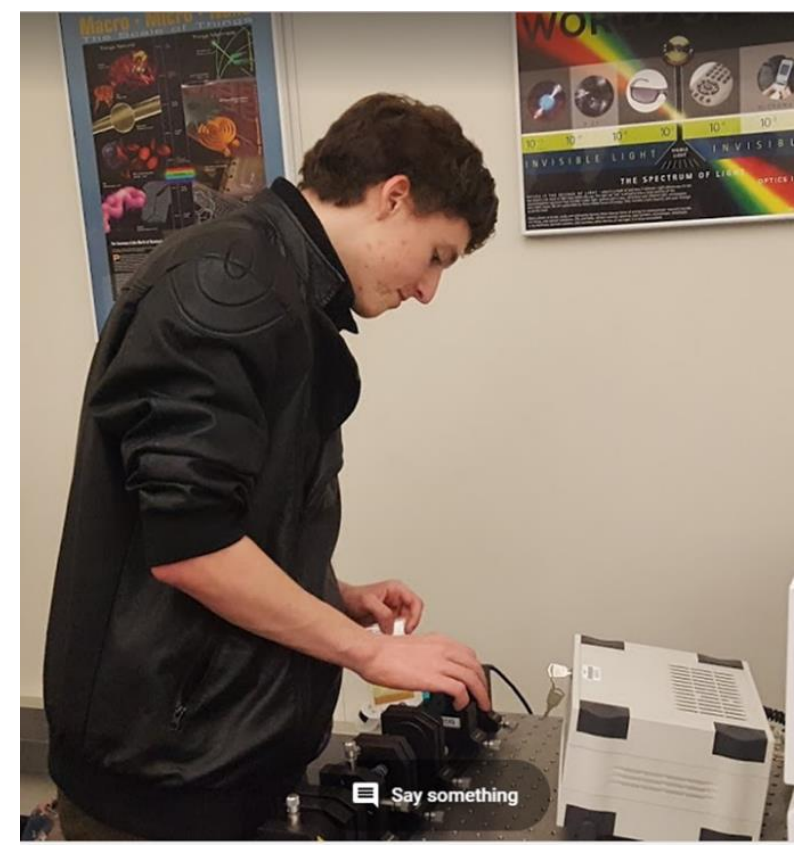

Fig. 10 is a photograph of Stefan setting up the Nd:YAG laser system. The system is not yet activated, or he would be wearing his laser safety glasses. 
The benefits of having the educational kits in class at IVC did not stop once I graduated from the program. When I subsequently worked as a lab technician for the program, these kits helped a lot when giving explanations for technical concepts to students. For example, if a group of students wants to learn more about laser cavity alignment for the HeNe laser kit, getting the kit up to that point in alignment isn't that difficult to do. From there I can show the alignment and all the tools I use and reset the kit by randomly turning the alignment knobs for each part. These kits are critical for our program to teach students alignment techniques that are used constantly in industry.

\section{Gabriel's Experiences and Perspectives on the Educational Aspects of the Kits}

Before I began taking the Laser Technology courses at IVC, I knew very little about light; I knew even less about lasers. My educational situation was unique. I heard about the four-course certificate program a year before transferring to University of California, Irvine. Since I hoped to achieve the certificate within three semesters, an exception was made for me to enroll in the second course of the series, Fundamentals of Photonics, before I took the Fundamentals of Light course, the first in the series. Therefore, I was entering a class with students who were either one class ahead of me or who were coming from industry jobs. Though I was tempted to feel discouraged by my lack of background knowledge and experience, hearing Dr. Brian Monacelli's first few lectures covering laser power classifications and safety, laser theory, and laser parts fascinated me and inspired me to press on. I set my mind to becoming the best laser technician I could learn to be in that one year; and if I didn't understand all the concepts at first, I would at least become fluent and excel in the technical aspects of the course.

\subsection{My Experience with the open-cavity HeNe laser}

The first HeNe laser I held in my hands was not an open-cavity HeNe, but rather a black cylindrical tube that looked like a supersized laser pointer - at least that's the only way I could describe it my second week in class. My lab team was using it to make a wavemeter. I knew that electricity went in one way (as the input), and I knew that a red beam came out the other (as the output) - but what happened in between was a mystery. To use a term from circuit analysis, it was a "black box" to me. In the successive lectures, Dr. Monacelli introduced the various components which make up a laser, such as the Excitation Mechanism, Gain Medium, High-Reflective Mirror, and Output Coupler. He also stressed the differences between spontaneous and stimulated radiation emission. These parts and concepts were excellently illustrated in drawings and diagrams on the presentation slides; still, since photonics was such a new world to me, I had no context with which I could process these ideas or connect them to anything I had experienced in real life. To see is to believe; to touch is to know. Yet, at this point, I still had no belief, for I had not seen the inside working components of a laser with my own eyes. I had no intimate knowledge of lasers either, for I had not yet tuned or aligned one with my own hands. Being both a visual and inquisitive learner, I needed a way to engage the laser.

Thankfully, IVC had a way to make this happen, and I didn't have to wait long to see the instrument that provided the solution. In week 5 of the course, I was introduced to the open-cavity HeNe laser, which was at the time being manufactured by the PI miCos Campus Educational department. The lab objective for the week was 
simple: assemble the parts of the HeNe laser and make it lase. That night I worked on the laser for an hour or two, but I could not produce any stimulated emission of radiation. However, something far more important was stimulated that night: my mind. As I read through the manual and examined the various components piece-by-piece, it was like I was simultaneously connecting the concepts together in my mind. The components were no longer blocks and ovals on a screen; now they had taken on three-dimensional shape. As I turned the key on the electronics controller to the 'on' position, causing the helium-neon gas trapped in the tube to spontaneously emit a reddishorange hue, it was like a lightbulb went on in my head. This is what spontaneous emission looks like. And as I began tuning the various components using the green diode laser as a reference beam, it finally all started to make sense.

It wasn't until later in the semester that I experienced the thrill of getting the HeNe to lase with my own hands. I turned the knob on the output coupler with one final quarter-twist and a red beam appeared (seemingly out of nowhere) and was sent across the room as if by some unexplainable magic. Except, it wasn't magic, it was photonics; and it wasn't unexplainable anymore, for I had held the components, assembled it on the rail, and seen them work together just the way my professor had said. I finally believed, and that was just the beginning point. I became curious, so I investigated the shape of the mirrors, and realized it was that of a long-radius hemispherical design - the highly-reflective mirror being concave and the output coupler being plano. I remembered learning in class that this was one of the more stable resonant cavity designs. Furthermore, I read in the textbook that the ease of the amplification process was positively related to the volume of the gain medium. Seeing the size of the tube which contained the helium-neon light, I realized why this instrument was so perfect for training new technicians. These of course were simple observations, but they wouldn't have been possible had I only worked with a capsulated HeNe laser. Without the open-cavity HeNe to give me perspective, HeNe lasers would have remained 'black boxes'; but in the IVC classroom, the 'black box' was illuminated with light.

The HeNe laser became an icon to the class. It was the final test. Everyone knew when someone had aligned it, for it was always accompanied with a cheer and an applause. Presenting a challenge to both the freshman and already-employed industry technicians alike, the HeNe was the great leveler. By succeeding in making it work myself, I had joined the ranks of the elite - perhaps not in their level of employment, yet, but certainly in my confidence.

\subsection{My experience with the Nd:YAG laser kit}

The summer following my first semester in the program, I was chosen to take the part-time role as the IVC photonics lab manager. I was responsible for organizing the lab, finding use for old equipment, purchasing new equipment, and assisting the professors in making the lab projects better. Just before I officially started the job, another Campus Laser Education kit appeared in our lab; lent to our lab by the same generous company. Thus, entered the Nd:YAG open-cavity laser kit, the new worthy challenger of our students. My first impression was that it contained a lot more components than the HeNe laser kit. I also learned that it emitted invisible 1064-nm light. I had not previously worked often with infrared lasers, but despite its daunting looks, I dared to make it work.

As I read the manual, I began to understand that the 808-nm diode laser was being used as both the alignment laser and the excitation mechanism. I recalled from my earlier Fundamentals of Photonics class the 
difference between electrical and optical excitation. This was one difference I was able to discern between the HeNe and Nd:YAG. The other more apparent difference was the lack of a long, lit, gas-filled tube. Where was the gain medium? This is when I noticed a five-axis mount holding a small crystal the size of a nail head. Of course, this is a solid-state laser - now I see! But where is the resonant cavity? This question confused me for a short time, until I realized that one side of the Nd:YAG crystal was coated, making it double as one of the mirrors of the oscillator. Very interesting. At that point everything began to fall into place. I needed to optically shape the beam from the diode laser using a collimator and a lens, then focus the beam into the Nd:YAG crystal, and then align the crystal with the output coupler to create the amplification necessary for lasing. However, since it was infrared light I was dealing with, I needed some way to measure it; so, I set up a detector and power meter, took note of the starting power measurement, and then ever so slightly adjusted the cavity mirrors until I saw the power reading begin to rise. I had done it! After optimizing the output power of the Nd:YAG, I had one last step: I inserted and aligned the frequency-doubling potassium titanyle phosphate (KTP) crystal inside the cavity via another mount. It immediately output 532-nm green light. Seeing this was pure delight. Figure 11 shows a photograph of this Nd:YAG Laser kit and Figure 12 is a diagram and descriptions of all the various components.

\section{eLas}

\section{Diode Pumped Nd:YAG Laser (CA-1230)}

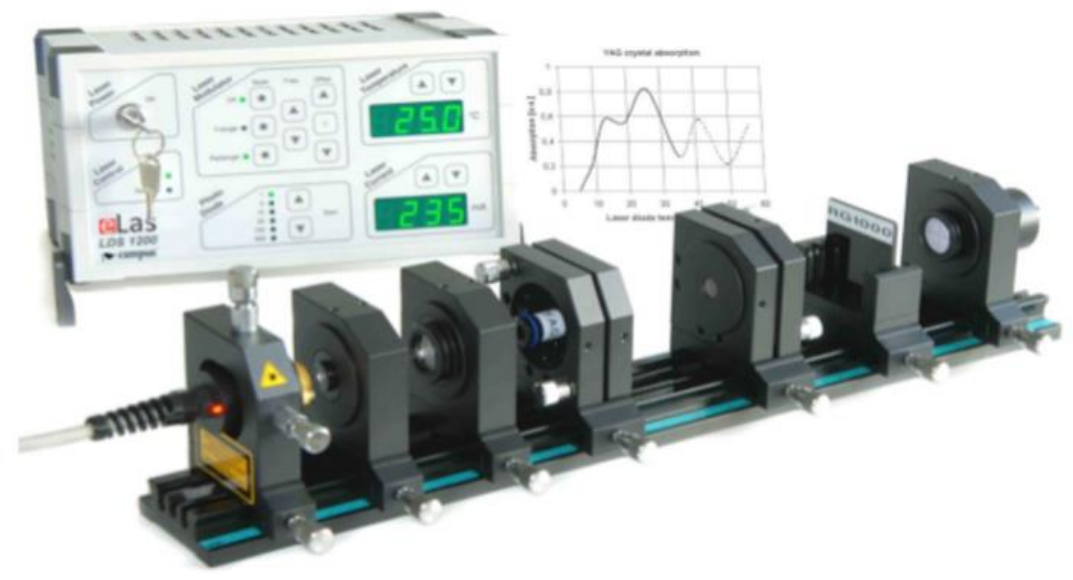

Fig. 11 shows a photograph of the eLas Nd:YAG Laser kit with of all the various components. 


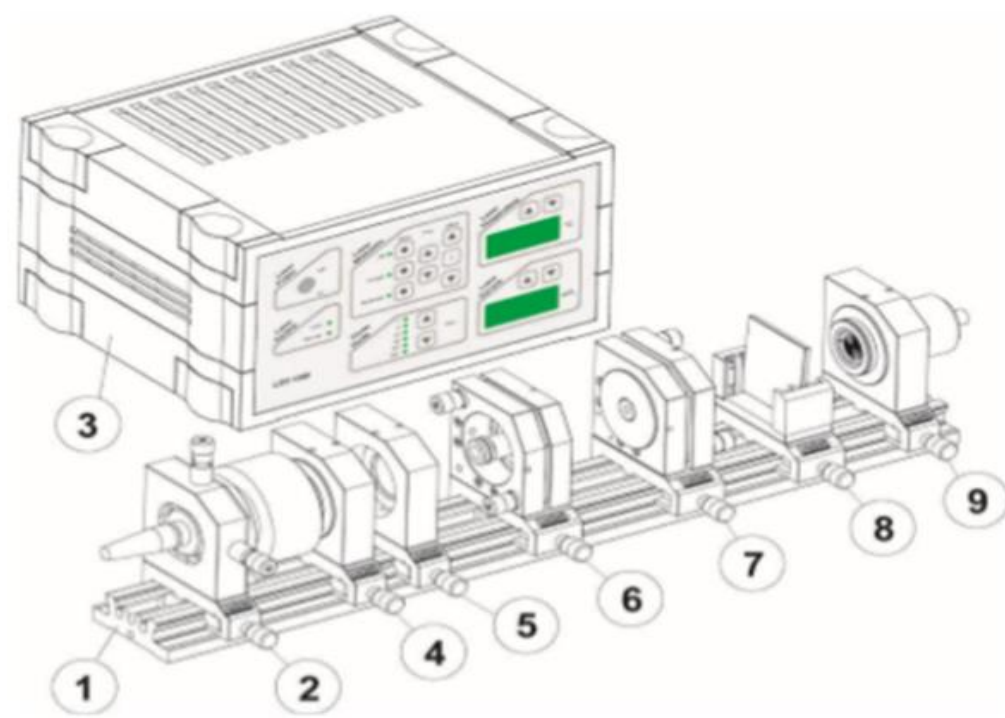

The Diode Pumped Nd:YAG laser kit includes:

1 Flat rail $500 \mathrm{~mm}$ with scale

2 Laser diode $450 \mathrm{~mW}$ with thermo-electric cooling in $\mathrm{X}$-Y adjustment holder on carrier

3 Microprocessor controlled laser diode supply LDS 1200 with thermo-electric controller, integrated current modulation and photo diode signal amplifier

4 Pump diode beam shaping optics in holder on carrier

5 Pump diode beam focusing optics in holder on carrier

$6 \mathrm{Nd}: \mathrm{YAG}$ crystal with mount in adjustment holder on carrier, $\mathrm{Nd}: \mathrm{YVO}_{4}$ crystal optionally available

7 Laser output mirror $(\mathrm{R}=98 \%)$ with mount in adjustment holder on carrier

8 Filter holder for three filter plates on carrier with filter RG1000

9 Si-PIN photo detector, spectral range $400 \mathrm{~nm}$ - $1100 \mathrm{~nm}$ in holder on carrier with adjustment target

10 Set of three BNC cables (not shown)

11 Infrared converter card $800-1600 \mathrm{~nm}$ (not shown)

12 Optics cleaning kit

13 Comprehensive user manual (not shown)

Fig. 12 shows a diagram with descriptions of all the various components. 
I graduated the Laser Technology program in Spring 2016, but my work at IVC was still funded through the summer. One of the final things I did while I was there was help us obtain via Perkins funds our very own $\mathrm{Nd}$ :YAG system, so that we could give back the one being lent to us. When it was finally time to say goodbye to the IVC Laser Lab, I was approached by Donn Silberman, who asked me if I would be interested in working for eLas Americas - a new startup company that was in the process of assuming the Campus Laser Education kits product line from PI miCos to continue to offer them to other schools. Of course, I took the job. Since my role covered that of sales and tech support, I needed to learn all that I could about the systems that we sold. To my surprise, there were a lot more than just the two systems I had experience with; there were kits for laser basics, optics fundamentals, laser metrology, fiber optics and telecommunications, and laser spectroscopy. In my first several months of working there, I received requests for quotation on almost all these systems, from physics professors, photonics instructors, and research supervisors across the nation.

The Nd:YAG system was by far the most popular of the eLas Americas offerings, so I focused a lot of my attention on learning its capabilities. The more I learned, the more I realized how powerful the Nd:YAG laser kit actually was as a hands-on teaching tool. When fitted with all the extra upgrades - including the frequency doubling crystal and the active and passive q-switches - the Nd:YAG laser kit actually has seven different operating modes, each one enabling a whole new set of experiments. Figure 13 lists these seven (7) modes of operation of the Nd:YAG laser education kit. When I was a student, I had no idea how much potential was built into this laser education kit. Because of my job at eLas Americas, I was able to continue working on the systems that brought me there in the first place, and I gained a whole new level of respect for them.

\section{Modes of Operation}

Diode Pumped Nd:YAG Laser (CA-1230)

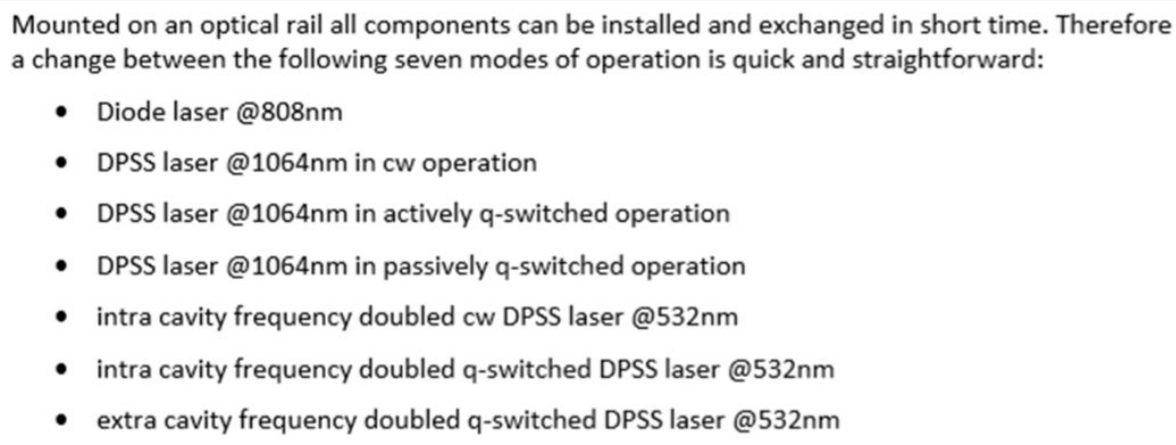

Fig. 13 lists seven (7) modes of operation of the Nd:YAG laser education kit. 
The Laser Technology program at IVC exists to raise up technicians to meet the industry demand. Speaking as one who graduated from this program and is now working in the field, the HeNe and Nd:YAG laser kits being used in this program and in other programs around the nation provide one of the most efficient and effective ways to teach students technical techniques, procedures, and safety. In my experience, these kits are essential to connecting theory with application and learning how laser components, electrical controllers, detectors and power meters can all work together to achieve an objective: a working laser. And whether the application is conducting research, performing a demonstration for a physics course, or doing such lab exercises as we did at IVC-spatial filtering, measuring beam width and beam divergence, coupling light into fiber optics, viewing the energy distribution on a high-resolution imager, testing detectors with various wavelengths, etc.--these lasers provide the light and the handson experiences students need to learn.

\section{Professors' Concluding Comments}

These open-cavity laser kits so successfully help instruct laser fundamentals to our students because they allow access to the inner components of otherwise sealed systems. As anyone who has even taken apart a computer or a television or even a toaster knows, disassembly and reassembly (particularly successful reassembly) of technical systems is a powerful and efficient way to learn about how they work and why different components are required. Commercial laser systems, like many modern electronics, are almost always fully integrated by the vendor, leaving their internal workings locked within a mysterious black box, as a then-uneducated Gabriel had initially observed. Granted, such sealed integrations are done for very good reason: lasers are extremely sensitive to misalignment, but their out-of-the-box monolithic nature does not help students understand why their alignment is so tenuous and how difficult it was to achieve and stabilize, particularly over strenuous operating conditions. Both the HeNe and the Nd:YAG open-cavity laser systems allow our students direct, visceral interaction with the individual components of these lasers. Many modular educational systems can be gimmicky, bearing little resemblance to their industrial counterparts; but these systems from eLas retain a lot of authenticity to a commercial laser, as both students agreed. The industrial-quality optomechanical components and the well-mounted, yet modular, optical components make these systems easily reconfigurable while creating a platform stable enough for laser operation.

After the fundamentals of laser systems are covered (starting with laser safety first!), the laboratory exercise that precedes alignment of these open-cavity lasers involves the inspection of broken, opened-cavity lasers. During this exercise, I can point to the components and discuss their purpose, but nothing can change. We even have an operational laser without a housing, so the Brewster windows are evident and the narrow bore of the pink glowing HeNe gain medium is pronounced, surrounded by the electrodes that provide the electronic excitation, but there are no adjustors. The students can take my word about laser operation, but no one in the room actually did anything to make the brilliant red laser beam emit from this laser's output coupler.

This demonstration is followed by a hands-on activity that uses a propagating laser beam to make parallel two glass windows by tipping and tilting the windows. In this activity, students are encouraged to seek the nuanced indicators of a quality optical alignment, such as interference of multiple reflected beams, and uniform diffraction of a beam within limiting apertures. After the students' sensitivities are tuned by this laboratory exercise that 
introduces alignment, they are encouraged to follow the detailed eLas instructions for aligning the mirrors to the gain media of the open-cavity lasers. Tedious, time-consuming, successful alignment of a laser cavity creates one of the most rewarding laboratory exercises available in modern education, as is evident by the exuberant exclamations when that vivid red or green laser beam finally propagates. Students are asked to document the steps they took to obtain laser output, as part of their grade for the course, and as a memo to their future selves, since their future employment may require them to remember the techniques they developed here. (And our graduates have proven this to be true!)

Students are also asked to make recommendations for improvement to this and all laboratory exercises. In fact, through the guidance of our students (particularly the two fellow authors of this paper), eLas has improved significantly its English-language descriptions of the sequences and parameters required to achieve laser alignment, including attention to the details mentioned above, such as the cleanliness of the optical components and the addition of the diverging lens to magnify the output. Awareness of the condition of the laser components plays well into our other hands-on curricula, particularly since the class that follows our Fundamentals of Photonics course (in which laser alignment is instructed), Quality Assurance of Precision Optics covers cleaning and inspection of precision optics. The students gain valuable, visceral experience with this hardware, and by reporting candid feedback, each student cohort then improves the experience for future classes.

\section{Conclusion}

In any technical discipline, it is important for students to interact with the technical product that is intended for industrial or commercial consumption. Too often, engineering programs teach without hardware, covering only the theory or the design steps of the complete engineering process. Student testimonials like those offered here demonstrate that we have an essential educational tool to offer the required interaction with the internal workings of laser systems. We expect that this tool will be required in our curricula for as long as ubiquitous laser technology influences our culture.

The IVC Laser Technology program partners with the Optical Society of Southern California, the Optics Institute of Southern California, the University of California, Irvine, industrial partners, and others to promote optics and laser education to Southern California residents and to recruit students. One of our outreach partners is Vital Link Orange County (http://www.vitallinkoc.org/ ). They provide many platforms in which to conduct educational optics outreach, such as their annual STEM \& the Arts Career Showcase. Figure 14 is a photograph of an author (Gabriel) demonstrating the components of the Nd:YAG laser kit to a family at this event. Figure 15 is a photograph of the joint exhibit booth showing how the Nd:YAG laser kit is part of a larger educational optics display showing attendees how many pieces of the local optics and laser community fit together. 


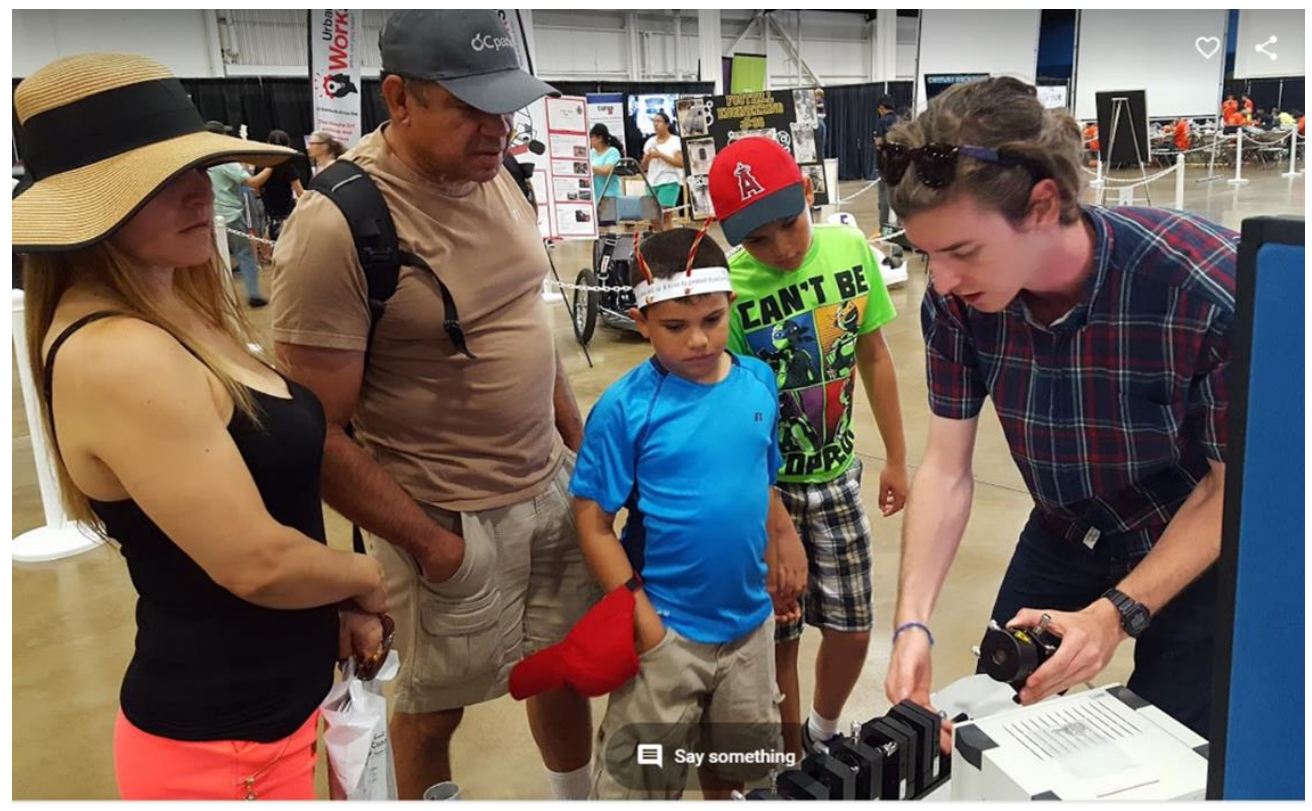

Figure 14 is a photograph of one of the authors (Gabriel) demonstrating the components of the Nd:YAG laser kit to a family at the Orange County Fair Grounds where the annual STEM \& the Arts Career Showcase takes place.

\section{eLas @ OSSC / OISC Outreach}

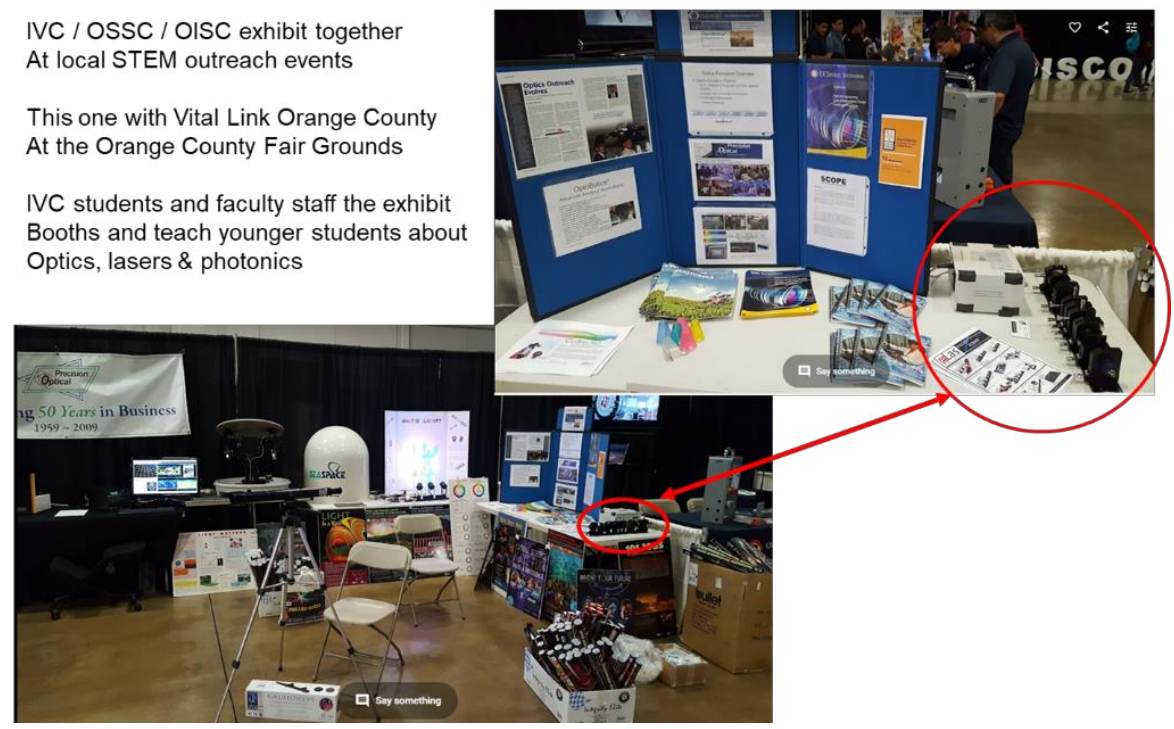

Figure 15 is a photograph of the joint exhibit booth showing how the Nd:YAG laser kit is part of a larger display showing attendees how many pieces of the local optics \& laser community fit together. 


\section{After thoughts}

Lead author Donn Silberman acknowledges that as a University of Arizona physics student in the early 1980s, he was fortunate to work as a student technician / assistant at the Optical Sciences Center for two years. One of his assignments was to gather educational experiments from various professors to be used in an Advanced Graduate Laboratory course. After graduating, while Mr. Silberman was a Laser Engineer for the Melles Griot Laser Products Division, he volunteered for Professor Sing Lee at the University of California, San Diego, Electrical Engineering Department, where he repaired educational laser systems and provided an open-cavity HeNe Laser from Melles Griot Laser Products. Continuing to give to the next and future generations is what we do, and we feel fortunate to be able to do so.

Proc. of SPIE Vol. 10741 107410G-17 